\title{
Impaired resolution of wheals in the skin prick test and low diamine oxidase blood level in allergic patients
}

\author{
Aneta Wagner ${ }^{1}$, Krzysztof Buczyłko², Hanna Zielińska-Bliźniewska ${ }^{1}$, Waldemar Wagner ${ }^{3}$ \\ ${ }^{1}$ Allergology and Respiratory Rehabilitation Department, Medical University of Lodz, Lodz, Poland \\ ${ }^{2}$ NZOZ Allergology Center, Lodz, Poland \\ ${ }^{3}$ Laboratory of Cellular Immunology, Institute of Medical Biology, Polish Academy of Sciences, Lodz, Poland
}

Adv Dermatol Allergol 2019; XXXVI (5): 538-543

DOI: https://doi.org/10.5114/ada.2019.89504

\begin{abstract}
Introduction: Histamine is the major mediator of IgE- and non-IgE-mediated allergic reactions upon allergen or hapten contact. Reduced histamine degradation capacity was associated with atopic eczema as well as with nonimmunological histamine intolerance. Higher blood serum histamine level concomitant with decreased intestinal diamine oxidase activity were observed in patients with food allergy.

Aim: To evaluate the relationship between patients' blood diamine oxidase (DAO) activity/histamine status and their reactivity to time-resolved histamine skin prick test in respect to vulnerability to allergic diseases.

Material and methods: Fifty-three patients were examined with skin prick tests (SPT) and patch tests for suspected presence of either IgE- or non-IgE-mediated allergy. All individuals were skin prick tested with histamine and the resolution of the wheal was monitored for $50 \mathrm{~min}$. Blood DAO activity and histamine concentration were measured with a radio-extraction radioimmunoassay.

Results: Time-resolved histamine skin prick testing revealed presence of wheals which were $35 \%$ larger in diameter in $47 \%$ of examined subjects at 20 min of the test. These patients exhibited significantly compromised time-course wheal resolution (wheal $\geq 3 \mathrm{~mm}$ at $50 \mathrm{~min}$ ) compared to a group of patients with the normal-rate of wheal resolution (wheal $=0 \mathrm{~mm}$ at $50 \mathrm{~min}$ ). Within a group of subjects exhibiting impaired wheal resolution, $61 \%$ of patients were diagnosed allergic compared to $50 \%$ in a group of patients with a normal rate of wheal resolution. Finally, allergic patients were characterized by a significantly lower DAO activity and higher histamine content compared to healthy subjects.

Conclusions: The results of this study indicate that patients with IgE- or non-lgE-mediated allergy are likely to have low DAO blood activity and may concomitantly suffer from histamine intolerance. Furthermore, our results suggest that allergic patients are more likely to develop an excessive SPT reaction. Our results emphasize caution in interpretation of the SPT results in allergic patients with diagnosed histamine intolerance or histamine/DAO activity imbalance.
\end{abstract}

Key words: diamine oxidase, histamine, allergy, wheal, skin prick tests.

\section{Introduction}

Food allergy is related to immune-mediated hypersensitivity to ingested allergens and histamine release from mast cells stores, whereas food-derived histamine is associated with non-allergic food hypersensitivity. Histamine (HI) scavenging repertoire in humans comprises the gastrointestinal barrier represented mostly by diamine oxidase (DAO, histaminase) and histamine $\mathrm{N}$-methyl transferase (HNMT) presented in the central nervous system and peripheral tissues [1]. DAO is a secretory protein, which operates in microvascular endo- thelium and inhibits the trans-epithelial permeation of exogenous histamine. Impaired degradation of histamine can follow a genetic or acquired impairment of the enzymatic function of DAO. The genes coding for DAO and HNMT enzymes are polymorphic and 7 diverse SNPs (single nucleotide polymorphisms) for DAO were significantly related with DAO serum activity [2]. The minor allele at rs2052129 was associated with a lower DAO mRNA expression for the homozygous minor allele, lower promoter activity and subsequently the increased risk of a reduced DAO activity [3]. Another mechanism

Address for correspondence: Waldemar Wagner PhD, Laboratory of Cellular Immunology, Institute of Medical Biology, Polish Academy of Sciences, 106 Lodowa St, 93-232 Lodz, Poland, phone: +48 4220933 97, e-mail: ownddiver@gmail.com Received: 26.04.2018, accepted: 19.06.2018. 
reducing DAO bioavailability might be related to medicines containing active agents against DAO. According to Leitner et al., common drugs or active ingredients such as cimetidine, verapamil, chloroquine and clavulanic acid are able to markedly reduce DAO activity by 50-90\% [3]. Moderate influence on DAO was caused by isoniazid and metamizole, acetyl cysteine and amitriptyline (> 20\%). Interestingly, another group of clinically used drugs - glycosaminoglycans can temporarily increase the DAO serum level [4]. Indeed, although DAO is expressed mainly in enterocytes and kidney epithelial cells, tissue-bound DAO can be released into circulation following stimulation with heparin with its serum half-life of about an hour [5]. Recently, the composition of fatty acids in the diet has also been shown to immensely affect DAO secretion [6]. Lymphatic intestinal DAO activity and protein secretion raised 4.5- and 3.5-fold, respectively following infusion of long-chain TG trilinolein (66\% of linoleic acid) in rats. Impaired degradation of histamine by DAO has serious clinical implications. Reduced activity of histaminase has been suggested to be the major reason for non-immunological histamine intolerance clinically manifested as diarrhoea, hypotension, headache, pruritus or flush [7]. Other experimental data also suggest that poor histamine tolerance might be associated with atopic subjects and history of asthma, eczema or rhinitis [8, 9]. Histamine intolerance (HIT) among allergic patients is being extensively studied as HIT symptoms are often concomitant with allergy and thus may hinder correct diagnosis. Recently, Kofler et al. proposed a 50 min' time-course histamine skin prick test procedure to discriminate HIT individuals against allergic patients [10].

\section{Aim}

In the current study we examined 53 subjects, nonallergic and allergic to pollen, mites or chemicals using histamine 50-skin prick test. Resulting SPT data were analysed in relation to patients' blood DAO activity/histamine content and their allergy status.

\section{Material and methods}

\section{Subjects}

The study group included 53 subjects, mean age 42 years with/without allergy. The study was approved by the Bioethics Committee of the Regional Medical Chamber in Lodz.

\section{Skin prick test (SPT) procedure}

IgE-mediated allergy was confirmed by SPT. Tests were performed according to the standard procedure with common allergens (pollens: hazel, alder, birch, grasses (mix), rye-grass, mugwort, mites: Dermatophagoides pteronyssinus, Dermatophagoides farinae, cat and dog epithelium, moulds: Alternaria alternata; Allergopharma $\mathrm{GmbH}$, Germany) with histamine dihydrochloride $(1.7 \mathrm{mg} / \mathrm{ml})$ as a positive control and SPT diluent ( $0.9 \%$ sodium chloride) as a negative control. The SPT results were read at $15 \mathrm{~min}$ and regarded as positive when the diameter of the wheal was $\geq 3 \mathrm{~mm}$. All patients confirmed that antihistamines were stopped no less than 7 days before testing.

\section{Histamine 50-skin prick test}

Histamine 50-skin prick test was performed according to Kofler et al. procedure with minor changes [10]. Briefly, patients' skin on the volar surface of the lower arm was pricked with histamine dihydrochloride $(1.7 \mathrm{mg} / \mathrm{ml})$ and diameter $(\mathrm{mm})$ of the developing wheal was measured after 15, 20 30, 40 and 50 min. On the basis of cut-off set at wheal size $\geq 3 \mathrm{~mm}$, at 50 min (best discriminating time-point and wheal size according to Kofler et al.), patients were classified into two groups: slow rate $\mathrm{HI}$ 50-wheal resolution group (HI 50-SPT $\geq 3 \mathrm{~mm}$ ) and high rate $\mathrm{HI} 50$-wheal resolution group $(\mathrm{HI}$ 50-SPT $<3 \mathrm{~mm}$ ).

\section{Patch testing procedure}

Non-lgE-mediated allergy was confirmed by patch tests. Tests were performed with European Baseline Series S-1000 containing 30 haptens (Chemotechnique Diagnostics, Sweden) according to the manufacturer's procedure. All participants confirmed that antihistamines were stopped no less than 7 days before testing and they were free of oral and topical steroids on the upper back area tested.

\section{Diamine oxidase and histamine analysis}

Peripheral blood samples were taken from fasting subjects. The blood histamine concentration and DAO activity were measured by Synevo laboratory (Synevo Studien Service Labor GmbH c/o IMD Institut für Medizinische Diagnostik Berlin-Potsdam GbR). Histamine was measured with an EIA assay (reference range $<75 \mathrm{ng} / \mathrm{ml}$ ). DAO activity was measured with a radio-extraction assay (reference range: 14-33 IU/ml) [11]. Briefly, determination of the enzymatic DAO activity was based on the conversion of radiolabelled-putrescine; a high affinity substrate for DAO. Then, the resulting radiolabel containing $\Delta^{1}$-pyrroline was extracted selectively from the matrix by a liquid extraction step. The amount of the $\Delta^{1}$-pyrroline detected with the scintillation counter was correlated with the patient's serum DAO activity.

\section{Statistical analysis}

The strength and direction of the linear relationship between these variables were evaluated via Pearson's correlation coefficient $(r)$. All calculations were performed 

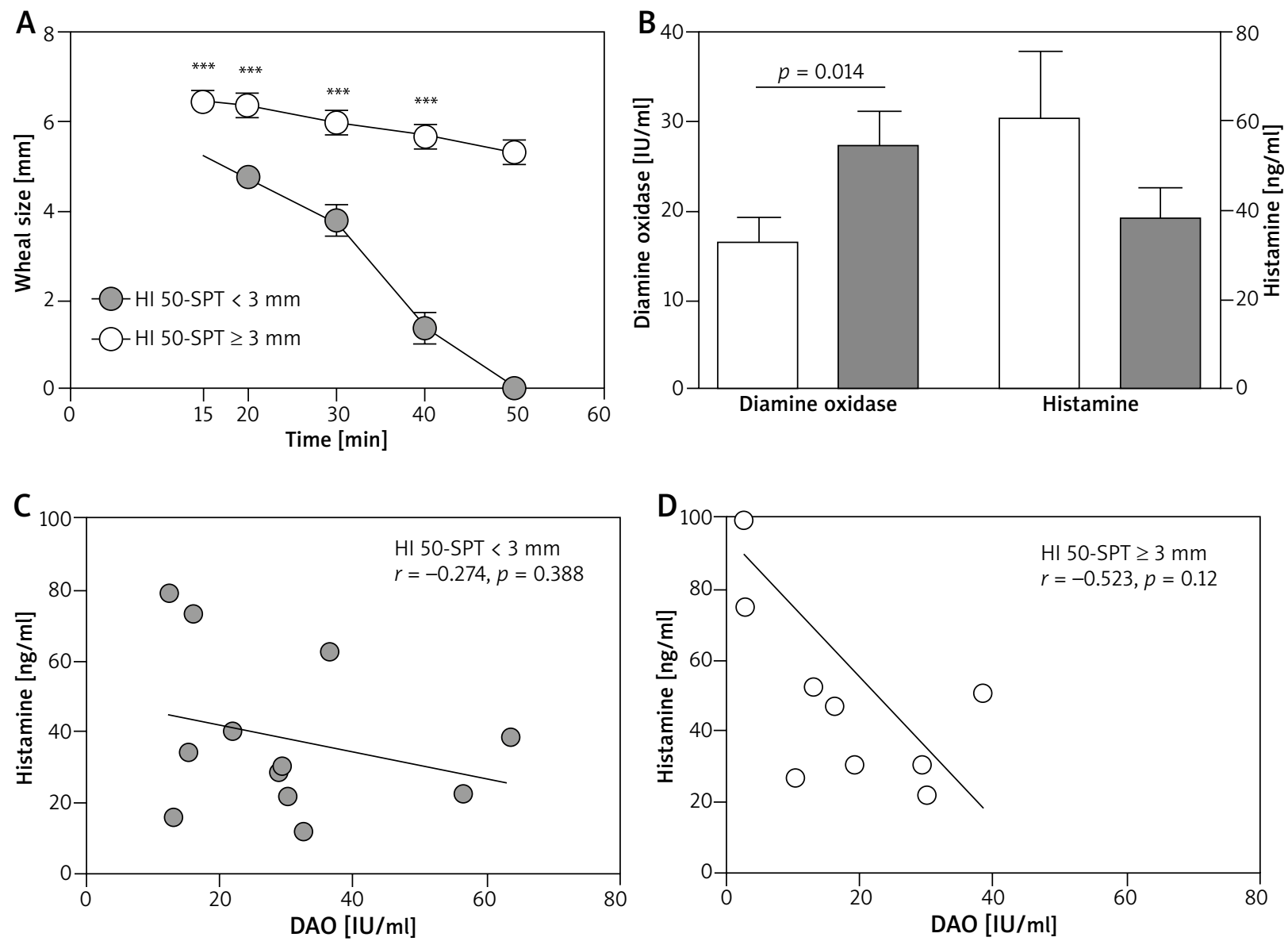

${ }^{\star \star \star} P<0.001$ indicates significant differences compared to the corresponding counterparts.

Figure 1. Blood diamine oxidase and histamine relationship in respect to skin reactivity and time-resolved histamine skin prick test. A - Time-course of histamine wheal resolution in HI 50-SPT $\geq 3 \mathrm{~mm}$ and HI 50-SPT $<3 \mathrm{~mm}$ groups of patients. B - Blood DAO activity and histamine concentration in HI 50-SPT $\geq 3 \mathrm{~mm}$ (white columns) and HI 50-SPT < $3 \mathrm{~mm}$ (grey columns) groups of patients. C, D - Pearson's correlation coefficient between DAO and histamine

with GraphPad Prism 5 at the assumed level of significance of $p \leq 0.05$.

\section{Results}

We studied the responsiveness of patients toward histamine using an enhanced SPT procedure (50 min' time-course skin prick test) in relation to their blood DAO activity and histamine (HI) concentration, and allergy status. On the basis of the results of this test, patients were classified into two groups: slow rate $\mathrm{HI}$ 50-wheal resolution group ( $\mathrm{HI} 50-\mathrm{SPT} \geq 3 \mathrm{~mm}$ ) and normal rate $\mathrm{HI}$ 50 -wheal resolution group (HI 50-SPT $<3 \mathrm{~mm})$. HI 50 SPT $\geq 3 \mathrm{~mm}$ group (47\% of patients) exhibited deeply compromised histamine-derived wheal resolution at each time point including the ones when SPT results are usually read in clinics (15-20 min) (Figure 1 A). At 15 and $20 \mathrm{~min}$, the wheals of the $\mathrm{HI} 50$-SPT $\geq 3 \mathrm{~mm}$ group were
$19 \%$ and $35 \%$ significantly larger than in the HI 50-SPT $<3 \mathrm{~mm}$ group $(p<0.001)$. Corresponding biochemical analysis of blood histamine degrading capacity showed that the HI 50-SPT $\geq 3 \mathrm{~mm}$ group had a significantly lower DAO activity $(16.33 \mathrm{IU} / \mathrm{ml}$ vs. $27.21 \mathrm{IU} / \mathrm{ml}, p=0.014)$ and a higher histamine concentration $(60.7 \mathrm{ng} / \mathrm{ml}$ vs. $38.33 \mathrm{ng} / \mathrm{ml}$ ) compared to the HI 50-SPT $<3 \mathrm{~mm}$ group (Figure $1 \mathrm{~B}$ ). Detailed analysis showed that all groups of examined patients $(r=-0.431, p=0.051)$ and each particular group of patients were characterized by an inverse correlation between DAO and histamine (Figures 1 C, D). The higher inverse correlation was observed for the $\mathrm{HI}$ 50-SPT $\geq 3 \mathrm{~mm}$ group $(r=-0.523, p=0.12)$ compared to the correlation coefficient for the HI 50-SPT $<3 \mathrm{~mm}$ group ( $r=-0.274, p=0.388)$.

On the basis of standard SPT with plants pollen, mites, moulds, cat and dog allergens and patch test with haptens we determined the allergy status of each subject within 

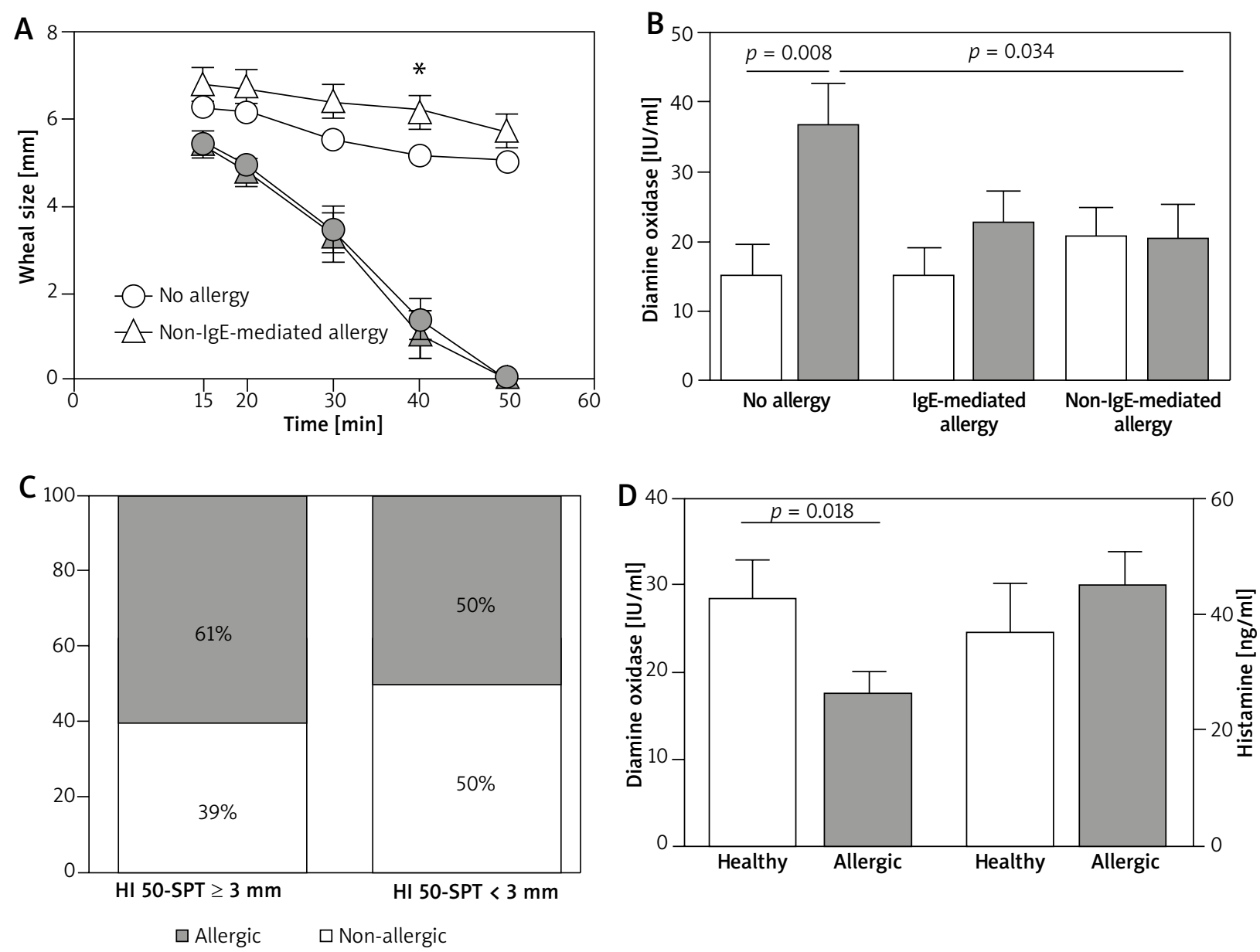

${ }^{\star} P<0.05$ indicates a significant difference compared to the corresponding counterpart.

Figure 2. Relationship between HI 50-SPT results and blood diamine oxidase activity in respect to patients' allergy status. A - Comparison of time-course histamine SPT between HI 50-SPT $\geq 3 \mathrm{~mm}$ (white figures) and HI 50-SPT < $3 \mathrm{~mm}$ (grey figures) groups of patients within healthy subjects (circles) and patients with non-lgE-mediated allergy (triangles). B - Comparison of DAO activity between HI 50-SPT $\geq 3 \mathrm{~mm}$ (white columns) and HI 50-SPT $<3 \mathrm{~mm}$ (grey columns) groups within healthy subjects, and patients with IgE- or non-IgE-mediated allergy. C - Incidence of the allergy in HI 50-SPT $\geq 3 \mathrm{~mm}$ (left column) and HI $50-S P T<3 \mathrm{~mm}$ groups (right column). D - DAO activity and histamine concentration in blood of healthy and allergic subjects

the examined group of patients. Interestingly, HI 50-SPT $\geq 3 \mathrm{~mm}$ patients with non-lgE-mediated allergy exhibited the most slow kinetics of histamine wheal resolution (Figure 2 A). Surprisingly, although the HI 50-SPT status corresponded with DAO activity within each subgroup of healthy persons (HI 50-SPT $<3 \mathrm{~mm}$ and high DAO activity; HI 50-SPT $\geq 3 \mathrm{~mm}$ and low DAO activity), these associations were not observed for groups of patients with lgE- and non-lgE-mediated allergy (Figure $2 \mathrm{~B}$ ). DAO activity in allergic groups of patients was low, irrespectively of their HI 50-SPT status. In the next step we analysed the incidence of diagnosed allergy in relation to the HI 50-SPT status. We found that within the HI $50-S P T \geq 3 \mathrm{~mm}$ group, $61 \%$ of subjects were allergic comparing to $50 \%$ in the $\mathrm{HI}$ $50-\mathrm{SPT}<3 \mathrm{~mm}$ group (Figure $2 \mathrm{C}$ ). Complimentary biochemical analysis of the DAO/histamine status revealed a significantly lower DAO activity and a slightly higher his- tamine concentration in blood of allergic patients comparing to healthy subjects (Figure 2 D).

\section{Discussion}

There is an increasing knowledge among clinicians concerning the relationship between interpretation of SPT results and skin reactivity. It is widely accepted that age, immunotherapy, habitat and menstrual cycle or even circadian rhythm and environmental exposure to irritants might affect the size of the wheal [12]. Furthermore, it appears that skin sensitivity has been increasing from year to year. Recently, the problem of histamine intolerance has been also raised as many symptoms of histamine intoxication mimics popular allergy symptoms [7, 13]. This has been recognised as a shortage of DAO expression in the intestinal barrier where diamine oxidase extracel- 
lularly catabolises the excessive amount of histamine to imidazole acetaldehyde, and protects amine from entering the systemic circulation [1]. Recently, the issue of DAO fluctuations in respect to suspected histamine intolerance and food allergy diagnostics has been raised [14]. In the following study steps we evaluated the influence of patients' histamine degrading capacity on SPT results in relation to their allergy status. According to Kofler et al., HIT could be distinguished from allergy by measurement of histamine-induced wheal resolution with a time-course SPT procedure [10]. The authors demonstrated that reading of wheal $\geq 3 \mathrm{~mm}$ at 50 min allows for discriminating for HIT with 79\% sensitivity and $81.3 \%$ specificity. In the present study we reported that a slow rate $\mathrm{HI}$ 50-wheal resolution group of patients (HI 50-SPT $\geq 3 \mathrm{~mm}$ ) exhibited deeply compromised wheal resolution at each time point of the test reading (15-50 min). Importantly, the size of wheals routinely read in clinics within 15-20 min of the test was significantly larger in the HI 50-SPT $\geq 3 \mathrm{~mm}$ group than in the HI 50-SPT $<3 \mathrm{~mm}$ group. Interestingly, we found an increased histamine concentration and concomitantly a significantly decreased DAO activity in blood of patients in the HI 50SPT $\geq 3 \mathrm{~mm}$ group. This group of patients also exhibited a close relationship between DAO and blood histamine content ( $r=-0.523$ vs. $r=-0.274$ of HI $50-$ SPT $<3 \mathrm{~mm}$ group) suggesting that their histamine catabolizing system might rely solely on DAO and operated close to its limits. Although these results are intriguing, they should be interpreted with caution due to the limited number of patients in each particular subgroup. Nevertheless, our observations are in line with the previous report indicating that such association of DAO and histamine is expected rather than a matter of coincidence $(r=-0.462)$ [14]. In the next step, we performed detailed analysis of $\mathrm{HI}$ 50-wheal resolution kinetics and DAO activity in each subgroup of healthy, IgE-mediated and non-IgE mediated allergic patients. We found that their HI 50-wheal resolution kinetics did not differ much from each other except for HI 50-SPT $\geq 3 \mathrm{~mm}$ groups between healthy and patients with non-lgE-mediated allergy (Figure $2 \mathrm{~A}$ ). Comparison of DAO activity results showed a similar association of the $\mathrm{HI} 50$-SPT $<3 \mathrm{~mm}$ group with high DAO activity and the HI 50-SPT $\geq 3 \mathrm{~mm}$ group with low DAO activity in the healthy group only, similarly to that previously noted for an unselected cohort (Figure 1 B). Interestingly, both allergic groups of patients exhibited a noticeable low DAO activity in each HI 50-SPT subgroup (Figure $2 \mathrm{~B}$ ). In other words, we observed the same rate of histamine wheal resolution in $\mathrm{HI} 50$-SPT $<3 \mathrm{~mm}$ groups within healthy and two allergic groups, although they significantly differed in blood DAO activity. Thus, in respect to these results the preliminary assumption that blood DAO activity regarded as a marker of intestinal DAO abundance might determine the extent of timeresolved skin prick test does not seem to be justified, at least for allergic patients. Consequently, exogenous histamine passing through the DAO barrier in the gastrointestinal tract and entering blood circulation could not be associated with the observed phenomenon of differences in the speed of wheal resolution. Collectively, our results are in line with the sparse scientific literature indicating HNMT as the dominating enzyme of histamine catabolism in the unstimulated skin (2.8-fold higher activity than DAO), although application of the proliferative stimuli may also result in DAO rise and concomitant histamine drop in the skin of the guinea pig $[15,16]$.

Contrary to a study of Kofler et al. aimed at HIT diagnosis in non-allergic patients with the use of HI 50-SPT, the results of our study indicated a significant contribution of slow histamine wheal resolution in both groups of patients with IgE- and non-IgE-mediated allergy. According to our study results, the concomitant slow histamine wheal resolution and allergy were diagnosed in $61 \%$ of patients (Figure 2 C) in comparison to $18 \%$ of patients in the Kofler study. Interestingly, subjects with a normal rate of histamine wheal resolution (HI 50-SPT $<3 \mathrm{~mm}$ group) were equally represented among allergic and healthy subjects. In the light of the recent findings, the concept of HIT, defined as altered histamine metabolism, may not explain all phenomena that lead to histamine imbalance affecting the development and course of the inflammatory reaction. Apart from food-derived histamine which constitutes mostly a systemic pool of the amine, the tissue-derived histamine, locally stored mostly by mast cells is even yet more important. Mast cells play a central role in inflammatory and immediate allergic reactions, sense environment for toxic substances and response to tissue injury through massive inflammatory mediators release including histamine. Recently, the mast cell activation syndrome (MCAS) characterized by abnormal mast cell sensitivity and excessive inflammatory mediator release has been described $[17,18]$. In patients with the secondary mast cell activation syndrome, mast cell activation is triggered by lgE-mediated stimuli (food, drug, Hymenoptera venom, or inhalant) or non-IgE-mediated stimuli (drugs, physical stimuli, stress, acute or chronic infections, venoms). According to Molderings et al., secondary MCAS is a fairly common disorder in Germany and may be an underlying cause of various clinical presentations (e.g. in subsets of patients with fibromyalgia and irritable bowel syndrome) and its incidence is likely to lie at least within the single-digit percentage range [19]. Thus, based on the results of our study and literature data, we cannot rule out the possibility that our observation of compromised histaminewheal resolution present in $47 \%$ of patients might be related in part to the mast cell hypersensitivity syndrome, although concomitant HIT also cannot be excluded from diagnostic considerations.

Apart from obvious HIT clinical symptoms resulting from DAO barrier impairment there is growing evidence 
of the association between subjects' histamine degrading capacity and diagnosed allergy. Such evidence for a reduced histamine degradation capacity was found in a subgroup of patients with atopic eczema [9] and atopic urticaria [8]. Furthermore, the latest study has shown that a genetic variation within the histamine pathway may be associated with allergic and non-allergic asthma phenotypes [20]. Indeed, we found that 55\% of subjects in the study represented allergic patients (with IgE- or non-lgEmediated allergy) with a significantly reduced DAO activity in blood compared to healthy ones (Figure $2 \mathrm{D}$ ).

\section{Conclusions}

Thus, according to collected literature data and own study results, we may tentatively suggest that allergic patients are more likely to develop an excessive SPT reaction. Particular caution should be taken during interpretation of SPT results in patients with diagnosed nonIgE-mediated allergy due to the possible occurrence of deeply compromised wheal resolution at 20 min of the test. Although, a positive control of histamine in the SPT procedure is a must, the degree of the tested allergen reaction might be erroneously overrated in diagnosed subjects. Finally, the results of this study indicate that patients with IgE- or non-IgE-mediated allergy are likely to have concomitant low DAO blood activity and possibly histamine intolerance symptoms. Further studies are needed that include larger numbers of subjects, especially in each allergic patients subgroup in order to elucidate the differences in the mechanism underlying differences in the SPT course and involvement of histamine metabolic pathways in the development of a particular allergy disease.

\section{Conflict of interest}

The authors declare no conflict of interest.

\section{References}

1. Fogel WA, Lewinski A, Jochem J. Histamine in food: is there anything to worry about? Biochem Soc Trans 2007; 35 : 349-52.

2. Maintz L, Yu CF, Rodríguez E, et al. Association of single nucleotide polymorphisms in the diamine oxidase gene with diamine oxidase serum activities. Allergy 2011; 66: 893-902.

3. Leitner R, Zoernpfenning E, Missbichler A. Evaluation of the inhibitory effect of various drugs/active ingredients on the activity of human diamine oxidase in vitro. Clin Transl Allergy 2014; 4 (Suppl 3): P23.

4. Klocker J, Perkmann R, Klein-Weigel P, et al. Continuous administration of heparin in patients with deep vein thrombosis can increase plasma levels of diamine oxidase. Vascul Pharmacol 2004; 40: 293-300.

5. Schwelberger HG. The origin of mammalian plasma amine oxidases. J Neural Transm 2007; 114: 757-62.

6. Ji Y, Sakata Y, Li X, et al. Lymphatic diamine oxidase secretion stimulated by fat absorption is linked with histamine release. Am J Physiol Gastrointest Liver Physiol 2013; 304: G732-40.

7. Wöhrl S, Hemmer W, Focke M, et al. Histamine intolerancelike symptoms in healthy volunteers after oral provocation with liquid histamine. Allergy Asthma Proc 2004; 25: 305-11.

8. Pollock I, Murdoch RD, Lessof MH. Plasma histamine and clinical tolerance to infused histamine in normal, atopic and urticarial subjects. Agents Actions 1991; 32: 359-65.

9. Maintz L, Benfadal S, Allam JP, et al. Evidence for a reduced histamine degradation capacity in a subgroup of patients with atopic eczema. J Allergy Clin Immunol 2006; 117: 1106-12.

10. Kofler L, Ulmer H, Kofler H. Histamine 50-skin-prick test: a tool to diagnose histamine intolerance. ISRN Allergy 2011; 2011: 353045.

11. Volker von Baehr. Ihr labor fur immunologische SpezialDiagnostik. Source: www.inflammatio.de/fileadmin/user_upload/inflammatio/Flyer_Broschueren/FAQ_Fragen_aus_ der_Laborpraxis_11_2017.pdf

12. Möller C. Histamine and its relation to allergens in the skin prick test. Int Arch Allergy Immunol 2015; 166: 241-2.

13. Manzotti G, Breda D, Di Gioacchino M, Burastero SE. Serum diamine oxidase activity in patients with histamine intolerance. Int I Immunopathol Pharmacol 2016; 29: 105-11.

14. Pinzer TC, Tietz E, Waldmann E, et al. Circadian profiling reveals higher histamine plasma levels and lower diamine oxidase serum activities in $24 \%$ of patients with suspected histamine intolerance compared to food allergy and controls. Allergy 2018; 73: 949-57.

15. Cho HJ, Cho SI, Kim HO, et al. Lack of association of plasma histamine with diamine oxidase in chronic idiopathic urticaria. Ann Dermatol 2013; 25: 189-95.

16. Fogel WA, Swiderska M, Maslinski C. Histamine catabolism in proliferating skin. Agents Actions 1989; 27: 224-6.

17. Afrin LB. Presentation, diagnosis, and management of mast cell activation syndrome. In: Mast Cells: Phenotypic Features, Biological Functions and Role in Immunity. Murray DB (ed). Nova Science Publishers, Inc. 2013.

18. Akin C. Mast cell activation syndromes. J Allergy Clin Immunol 2017; 140: 349-55.

19. Molderings GJ, Haenisch B, Bogdanow M, et al. Familial occurrence of systemic mast cell activation disease. PLoS One 2013; 8: e76241.

20. Anvari S, Vyhlidal CA, Dai H, Jones BL. genetic variation along the histamine pathway in children with allergic versus nonallergic asthma. Am J Respir Cell Mol Biol 2015; 53: 802-9. 